\title{
柴北缘西段超高压地体折返过程中的部分熔融: 榴辉岩及其退变产物中的线色脉体研究
}

陈丹玲"，曹玉亭，刘良

大陆动力学国家重点实验室, 西北大学地质学系, 西安 710069

* 联系人, E-mail: dlchen@nwu.edu.cn

2013-05-27 收稿, 2013-06-14 接受, 2013-07-29 网络版发表

国家重点基础研究发展计划(2009CB825003)和国家自然科学基金(41072051)资助

\begin{abstract}
摘要 岩石学、地球化学和同位素年代学研究表明, 柴北缘西段锡铁山和胜利口地区的 榴辉岩/退变榴辉岩中的浅色脉体是超高压地体折返过程中榴辉岩发生部分熔融的结果. 浅色脉体主要由粗晶斜长石组成, 包绕或切穿榴辉岩/退变榴辉岩体. 脉体中的锆石呈自 形柱状形态, 具有明显岩浆成因震荡环带结构和包体矿物组合, 定年结果得到脉体的形 成时代分别晚于寄主榴辉岩/退变榴辉岩的峰期变质时代约 20 30 Ma，与寄主岩石麻粒岩 相退变质时代一致, 指示熔融作用发生在折返过程的麻粒岩相退变质阶段. 浅色脉体具 有高 $\mathrm{Si}, \mathrm{Al}, \mathrm{Ca}, \mathrm{Na}, \mathrm{Sr}, \mathrm{Pb}$ 和 LREE 而低 $\mathrm{Mg}, \mathrm{Fe}, \mathrm{K}, \mathrm{Y}, \mathrm{Yb}$ 和 HREE 的英云闪长岩的地球化 学特征, 䵢穷石和绿辉石的脱水分解是触发超高压榴辉岩发生部分熔融形成这些浅色脉 体的主要机制.
\end{abstract}

\section{关键词}

浅色脉体

榴辉岩/退变榴辉岩

部分熔融

锆石年代学

柴北缘
实验岩石学和天然样品的大量研究表明, 含水 矿物的分解和名义上无水矿物中水的释放在超高压 变质岩石折返过程的部分熔融中扮演着很重要的角 色 ${ }^{[1 ~ 8]}$. 熔融产生熔体的成分和量的多少主要取决于 含水矿物类型和温压条件 ${ }^{[2]}$. 多硅白云母、褐帘石、 䁫帘石和角闪石是导致超高压变质岩石发生部分熔 融最主要的几种含水矿物 ${ }^{[1,2,4 \sim 6,9]}$. 一般来讲, 多硅白 云母的脱水熔融多形成高 $\mathrm{Si}$ 、高 $\mathrm{K}$ 并富含 $\mathrm{Rb}$ 和 $\mathrm{Ba}$ 的长英质熔体, 是目前 HP-UHP 变质地体中富钾高 硅的花岗质脉体和构造后高钾花岗岩的主要形成机 制 ${ }^{[1,4 \sim 6,8,10,11]}$; 黝帘石的部分熔融可以形成高 $\mathrm{Sr}$, LREE, $\mathrm{Pb}$ 的英云闪长质和奥长花岗质熔体 ${ }^{[2]}$; 绿辉石的部 分熔融产生高 $\mathrm{Na} / \mathrm{K}$ 和高 $\mathrm{Ca}$ 的熔体 ${ }^{[12]}$; 角闪石的部 分熔融由于发生在斜长石的稳定域, 因而它所产生 的熔体成分中 $\mathrm{Ca}$ 和 $\mathrm{Sr}$ 的含量会降低，同时会出现明 显的 $\mathrm{Eu}$ 的负异常 ${ }^{[2]}$. 对基性榴辉岩的部分熔融实验
表明 ${ }^{[2]}$, 随着压力的升高, 实验产生的原始熔体的成 分在 $1.0 \mathrm{GPa}$ 时为英云闪长质, $1.5 \mathrm{GPa}$ 时为英云闪 长-奥长花岗质, $2.1 \sim 2.7 \mathrm{GPa}$ 时为石英二长质, 当压 力升高到 $3.2 \mathrm{GPa}$ 时产生典型花岗岩浆. 因此, 详细 研究高压-超高压地体中各种浅色脉体的物质组成、 形成条件和形成时代, 对于理解超高压变质地体俯 冲和折返过程中的变质脱水和部分熔融发生的温压 条件、元素的活化和迁移特征, 以及与同构造岩浆作 用的关系等具有重要意义. 与多数已报道的超高压 变质地体中主要以富钾硅质脉体不同, 在柴北缘超 高压变质带西段锡铁山超高压榴辉岩和胜利口地区 退变榴辉岩中产出的浅色脉体主要以斜长石为主, 不含或含极少量的钾长石. 本文主要介绍这两个地 区超高压地体中浅色脉体的物质组成和形成时代, 并通过与寄主榴辉岩/退变榴辉岩的对比, 探讨其形 成过程和机理.

引用格式: 陈丹玲, 曹玉亭, 刘良. 柴北缘西段超高压地体折返过程中的部分熔融：榴辉岩及其退变产物中的浅色脉体研究. 科学通报, 2013, 58: 2209-2214

Chen D L, Cao Y T, Liu L. Partial melting of UHP terranes in the western segment of the North Qaidam during exhumation: constraints from studies of leucocratic veins within eclogite/retrograde eclogite. Chin Sci Bull, 2013, 58: 2209-2214, doi: 10.1360/972013-612 


\section{1 区域地质背景与野外特征}

柴北缘超高压变质带呈狭长带状夹于祁连地块 和柴达木地块之间, 是一个形成于早古生代的典型 大陆型俯冲碰撞构造带 ${ }^{[13 ~ 23]}$, 从东到西主要由都兰、 锡铁山、胜利口和鱼卡 4 个超高压地体组成(图 1). 锡 铁山和胜利口超高压地体位于该带的中西部, 区内 榴辉岩或石榴石橄榄岩呈透镜状产于围岩片麻岩之 中. 锡铁山超高压地体主要由超高压榴辉岩和片麻 岩组成. 最新研究表明, 锡铁山榴辉岩和围岩-含蓝 晶石/矽线石石榴石黑云母片岩经历了相同的变质 $P-T$ 轨迹, 在 440 460 Ma 经历了陆壳深俯冲超高压变 质 ${ }^{[22,23]}$, 随后在 422 425 Ma 经历折返退变质改造 ${ }^{[23]}$. 胜利口超高压地体主要由超高压石榴橄榄岩、纯橄 岩、石榴辉石岩、退变榴辉岩和片麻岩组成. 年代学 研究得到超高压变质作用的时代为 $423 \pm 5 \mathrm{Ma}^{[15]}$ 或 $429 \pm 2 \mathrm{Ma}^{[25]}$, 并经历了 $397 \pm 6 \mathrm{Ma}$ 的后期热事件叠 加 ${ }^{[15]}$. 退变榴辉岩也被称为基性麻粒岩, 张建新等 人 $^{[24]}$ 在该岩石的锆石中曾得到 $448 \pm 3$ 和 $421 \pm 5 \mathrm{Ma}$ 两 组年龄, 认为是麻粒岩发生高压和中低压麻粒岩相 变质的年龄. 由于该岩石石榴石中绿辉石包体的普 遍存在 ${ }^{1)}{ }^{[25]}$, 我们称之为退变榴辉岩. Zhang 等人 ${ }^{[26]}$
随后又给出该岩石的围岩-含石榴石蓝晶石黑云母片 麻岩的变质和退变质年龄分别为 450 和 425 Ma.

野外观察发现, 锡铁山超高压榴辉岩和胜利口 退变榴辉岩中存在大量浅色脉体 (图 2). 在锡铁山榴 辉岩中, 这些浅色脉体包绕榴辉岩透镜体产出, 脉体 宽度从 $<1 \mathrm{~cm}$ 到几十厘米, 与榴辉岩同步变形, 局部 切穿榴辉岩体(图 2(a), (b)). 脉体矿物颗粒粗大且自 形程度高, 指示脉体形成在榴辉岩变质之后; 胜利口 地区退变榴辉岩中的浅色脉体宽度在 5 20 $\mathrm{cm}$, 变形 微弱(图 2(c)), 脉体与寄主岩石之间关系截然, 局部 与脉体接触部位石榴辉石岩发生强烈的角闪岩化, 变为石榴角闪岩(图 2(d)).

\section{2 浅色脉体的岩石学和地球化学特征}

锡铁山超高压榴辉岩和胜利口退变榴辉岩中的 浅色脉体主要由粗粒自形的斜长石和石英组成(锡铁 山：60\% 65\%斜长石+35\% 40\%石英; 胜利口：75\% $85 \%$ 斜长石 $+10 \%$ 15\%石英), 另含 $<1 \%$ 的黑云母+白 云母土角闪石土黑云母土钾长石 ${ }^{1)}{ }^{[27]}$. 岩石地球化学研 究显示, 浅色脉体具有高 $\mathrm{SiO}_{2}$ (胜利口和锡铁山浅 色脉体分别为 $66.92 \%$ 78.38\%和 $61.10 \%$ 65.77\%),

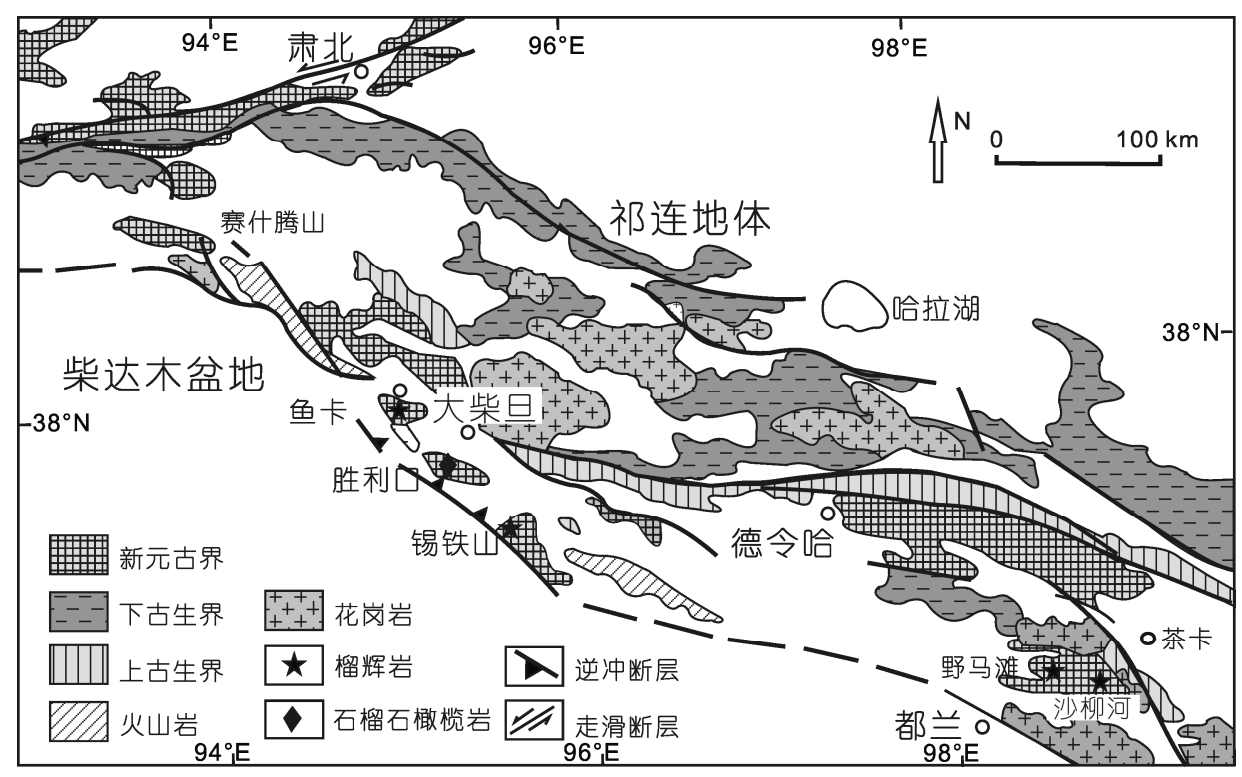

图 1 柴北缘高压-超高压变质岩带及邻区构造分区图 据文献[24]修改

1) Cao Y T, Liu L, Chen D L, et al. The felsic vein within the garnet pyroxenite from Shenglikou, North Qaidam: episodic fluid flow during the exhumation of the rock. Lithos, (in review) 

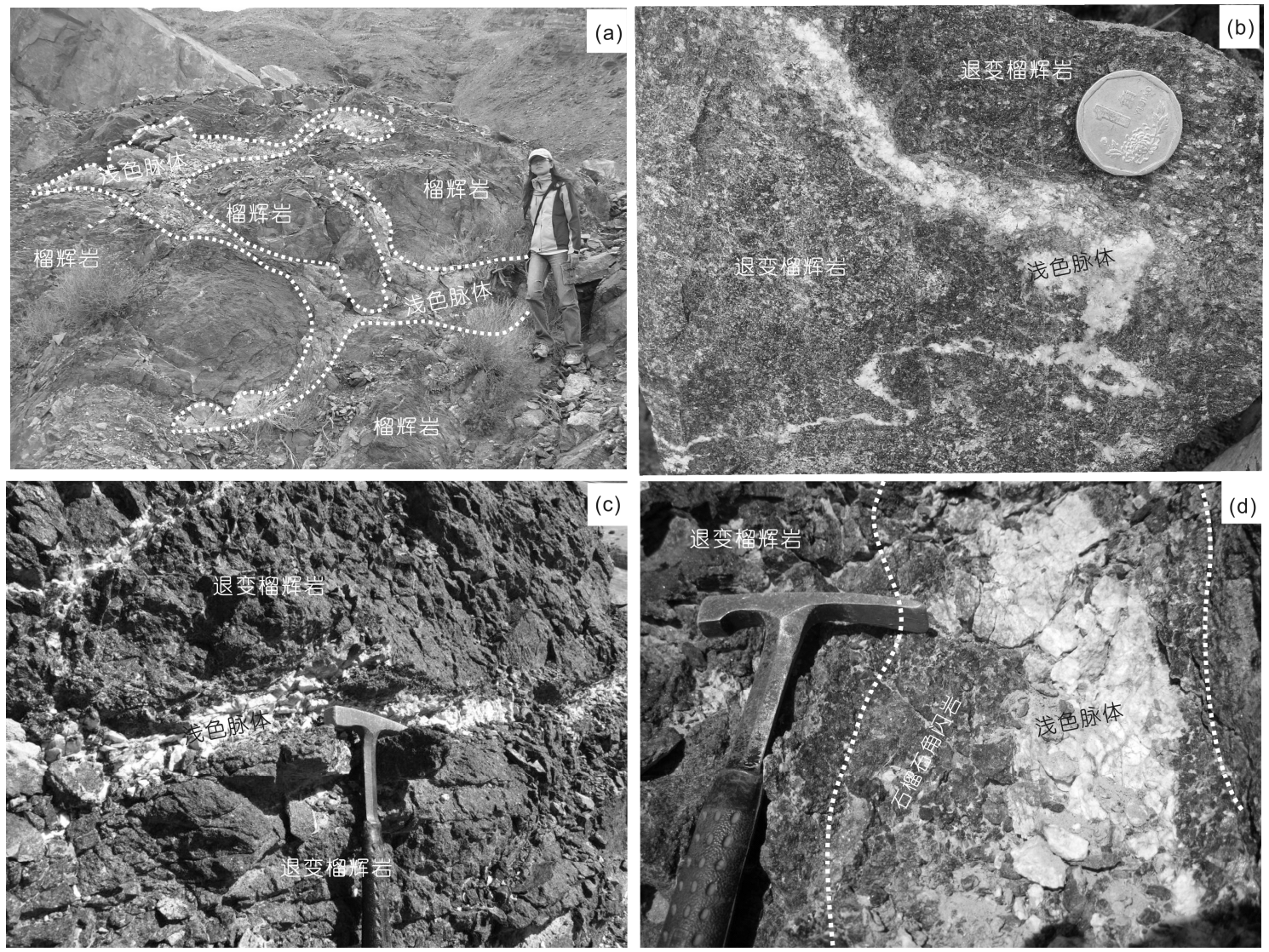

图 2 锡铁山榴辉岩和胜利口退变榴辉岩中浅色脉体的野外产状

(a) 锡铁山榴辉岩体中不规则状浅色脉体, 将榴辉岩分割成多个透镜状体, 脉体与围岩不连通; (b) 榴辉岩中的浅色细脉尖灭于榴辉岩体中; (c) 胜利口退变榴辉岩中的浅色脉体切过辉石岩体; (d) 胜利口退变榴辉岩与脉体的过渡带形成石榴石角闪岩

$\mathrm{Al}_{2} \mathrm{O}_{3}(20.32 \% \sim 23.18 \%$ 和 $12.46 \% \sim 20.60 \%), \mathrm{CaO}(4.48 \%$ 6.97\% 和 $3.21 \% \sim 5.23 \%), \mathrm{Na}_{2} \mathrm{O}(5.48 \% \sim 6.65 \%$ 和 3.77\% $6.45 \%$ )和低 $\mathrm{MgO}, \mathrm{FeO}, \mathrm{K}_{2} \mathrm{O}$ 和 $\mathrm{TiO}_{2}$ 的特征, 富集 $\mathrm{Sr}$, $\mathrm{Pb}$ 和 LREE，亏损 $\mathrm{Y}, \mathrm{Yb}, \mathrm{Nb}$ 和 HREE，并显示 LREE 富集、HREE 亏损、Eu 正异常明显的英云闪长岩的 特征 ${ }^{1)}{ }^{[27]}$ (图 3).

\section{3 锆石年代学}

分离自浅色脉体中的锆石均呈自形柱状形态, 内具振荡环带结构(图 4(a), (b)), 并具有轻稀土亏损、 重稀土明显富集的曲线特征 ${ }^{1)}{ }^{[27]}$. 锆石中的包体矿物 组合(斜长石+石英土白云母)也与脉体的矿物组合一 致, 与寄主榴辉岩或退变榴辉岩中锆石的特征明显 不同, 表明锆石是从脉体中直接结晶. 榴辉岩/退变 榴辉岩中的锆石多呈浑圆粒状形态, 其中锡铁山榴 辉岩锆石内部均匀无结构(图 4(b)), 含有石榴石、绿 辉石、金红石等榴辉岩相显微矿物包体, 具有低的稀
土元素含量和 $\mathrm{Th} / \mathrm{U}$ 比值, 以及 HREEs 平坦型、不具 $\mathrm{Eu}$ 负异常的稀土配分曲线特征 ${ }^{[27]}$. 胜利口退变榴辉 岩锆石内部显示核-幔-边或核-边结构(图 4(c)), 其中 核部具有较高的 REE 含量、HREEs 富集型稀土配分 模式、明显的 $\mathrm{Eu}$ 异常和较高的 $\mathrm{Th} / \mathrm{U}$ 比值, 为原岩岩 浆锆石残留; 幔部具有较低的 REE 含量和 Th/U 比 值、平坦的 HREEs 配分模式、无 $\mathrm{Eu}$ 负异常，表明形 成于榴辉岩相变质阶段; 边部可见到石榴石、斜长石 和单斜辉石的麻粒岩相包体矿物组合，具有低的 REE 含量和 HREEs 平坦型稀土配分模式，明显的 $\mathrm{Eu}$ 负异常和较低的 $\mathrm{Th} / \mathrm{U}$ 比值, 为麻粒岩相变质增生结 果 ${ }^{1)}$. LA-ICPMS 定年结果得到锡铁山榴辉岩的榴辉 岩相变质年龄和浅色脉体的形成年龄分别为 $458 \pm 2$ 和 428 $\pm 2 \mathrm{Ma}$ (图 4(a), (b) $)^{[27]}$; 获得胜利口退变榴辉岩 的原岩结晶年龄、榴辉岩相变质年龄及麻粒岩相退变 质年龄分别为 $909 \pm 6,444 \pm 4$ 和 420 $\pm 3 \mathrm{Ma}$ (图 4(d)), 获得长英质脉体的结晶年龄 $422 \pm 2 \mathrm{Ma}$ (图 4(c)). 其 

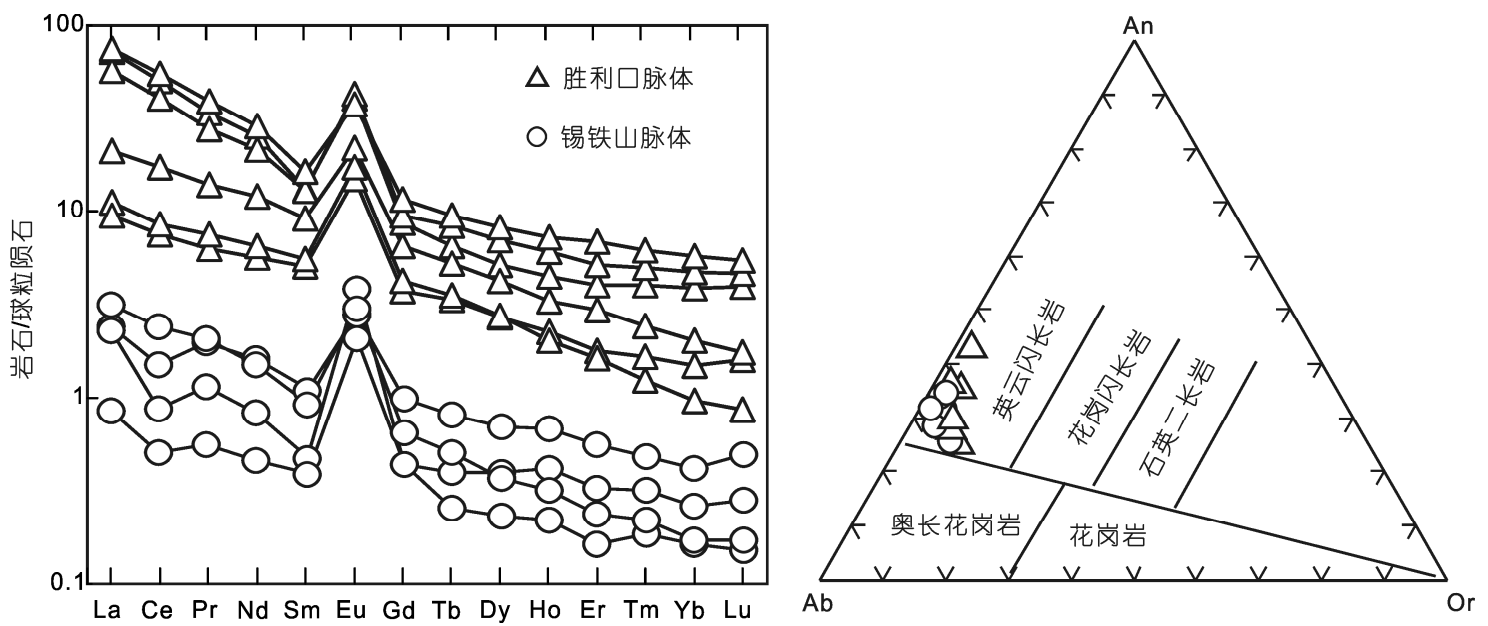

图 3 浅色脉体的球粒陨石标准化稀土配分模式图(a)和 Ab-An-Or 三角图解(b) 数据来自 1)和文献[27]
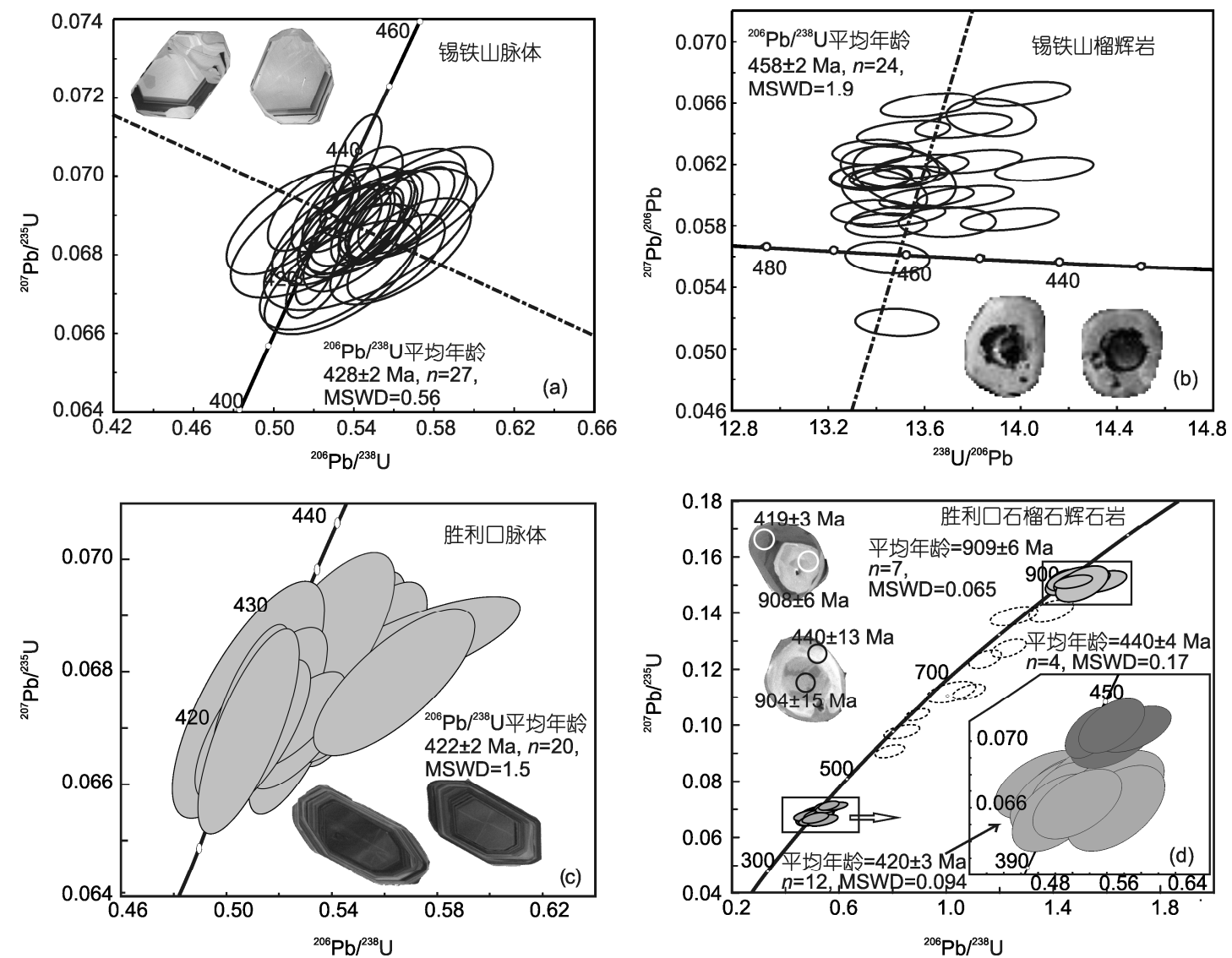

图 4 浅色脉体及其寄主岩石的锆石形貌和定年结果

数据来自 1)和文献[27]. (a), (b) 锡铁山榴辉岩和浅色脉体; (c), (d) 胜利口退变榴辉岩和浅色脉体

中退变榴辉岩中的榴辉岩相和麻粒岩相两期变质年 龄 $(444 \pm 4$ 和 $420 \pm 3 \mathrm{Ma})$ 与前人在该岩石中获得高压和 低压麻粒岩相变质年龄的数值 $(448 \pm 3 \text { 和 } 421 \pm 5 \mathrm{Ma})^{[24]}$
在误差范围内完全一致，只是两者的解释不同. 石榴 石中绿辉石包体的发现 ${ }^{1)[24]}$ 以及锆石无 $\mathrm{Eu}$ 异常的 HREEs 平坦型配分模式，表明 444 4 Ma (本文)或者 
$448 \pm 3 \mathrm{Ma}^{[24]}$ 应该代表榴辉岩相变质年龄. 另外, 本 次获得的 $420 \pm 3 \mathrm{Ma}$ (或 $421 \pm 5 \mathrm{Ma})^{[24]}$ 麻粒岩相退变 质年龄, 与脉体的结晶年龄 $(422 \pm 2 \mathrm{Ma})$ 一致.

\section{4 形成机制讨论}

锡铁山榴辉岩和胜利口退变榴辉岩中的浅色脉 体包绕或切穿榴辉岩/退变榴辉岩透镜体, 变形都很 弱, 且组成矿物自形程度高, 呈粗晶到巨晶形态, 显 示脉体形成于榴辉岩峰期变质之后. 定年结果得到 脉体的形成时代都晚于相应的寄主岩石的峰期变质 时代约 20 30 Ma, 而且与寄主岩石及区内麻粒岩相 退变质时代以及侵人到超高压地体中的埃达克质花 岗岩体的形成时代(428 430 Ma) ${ }^{[26,28,29]}$ 一致, 说明脉 体形成于超高压地体的折返阶段, 并且可能与区内 I 型花岗岩的侵位相关 ${ }^{[27]}$.

目前在超高压地体中出现的浅色脉体以高钾硅 质脉体最为常见, 折返过程中多硅白云母的脱水熔 融被解释为主要的形成机制 ${ }^{[1,4 \sim 6,8,11,30]}$. 与此不同的
是, 柴北缘西段锡铁山榴辉岩及胜利口退变榴辉岩 中的浅色脉体总体显示了高 $\mathrm{Si}$ 富 $\mathrm{Ca}, \mathrm{Al}$ 和 $\mathrm{Na}$ 而贫 $\mathrm{K}$ 的英云闪长岩的地球化学特征(图 3(b)). 虽然我们不 能排除脉体的形成过程中可能存在矿物堆晶以及熔 体抽取等因素 ${ }^{[31]}$, 但这两地的脉体成分与基性榴 辉岩部分熔融产生熔体的实验结果 ${ }^{[2,32]}$ 和地质发 现 ${ }^{[33,34]}$ 吻合. 脉体中很低的 $\mathrm{K}$ 含量, 一方面排除了多 硅白云母部分熔融形成脉体的可能性，同时也可排 除榴辉岩和退变榴辉岩的围岩-花岗质片麻岩和含蓝 晶石多硅白云母石英片麻岩参与熔融的可能性. 浅 色脉体是榴辉岩/退变榴辉岩在折返过程中发生部分 熔融的结果. 脉体显示的富集 $\mathrm{Ca}, \mathrm{Sr}, \mathrm{Pb}$ 和 LREE，贫 $\mathrm{Y}$ 和 $\mathrm{Yb}$ 亏损 HREE 以及明显的 $\mathrm{Eu}$ 正异常的微量元 素特征与黝帘石脱水熔融形成熔体的特征一致 ${ }^{[2,9]}$, 说明超高压地体折返过程䵢窝石的脱水分解是导致 超高压榴辉岩发生部分熔融的主要机制; 脉体中 较高的 $\mathrm{Na}$ 含量, 说明在部分熔融过程中有绿辉石的 贡献.

\section{参考文献}

1 Schmidt M W, Vielzeuf D, Auzanneau E. Melting and dissolution of subducting crust at high pressures: The key role of white mica. Earth Planet Sci Lett, 2004, 228: 65-84

2 Skjerlie K P, Patiño Douce A E. The fluid-absent partial melting of a zoisite-bearing quartz eclogite from 1.0 to 3.2 GPa: Implications for melting in thickened continental crust and for subduction-zone processes. J Petrol, 2002, 43: 291-314

3 Wallis S, Tsuboi M, Suzuki K, et al. Role of partial melting in the evolution of the Sulu (eastern China) ultrahigh-pressure terrane. Geology, 2005, 33: 129-132

4 Hermann J, Spandler C, Hack A, et al. Aqueous fluids and hydrous melts in high-pressure and ultra-high pressure rocks: Implications for element transfer in subduction zones. Lithos, 2006, 92: 399-417

5 Zheng Y F. Fluid regime in continental subduction zones: Petrological insights from ultrahigh-pressure metamorphic rocks. J Geol Soc London, 2009, 166: 763-782

6 Auzanneau E, Vielzeuf D, Schmidt M W. Experimental evidence of decompression melting during exhumation of subducted continental crust. Contrib Mineral Petrol, 2006, 152: 125-148

7 Xia Q X, Zheng Y F, Zhou L G. Dehydration and melting during continental collision: Constraints from element and isotope geochemistry of low-T/UHP granitic gneiss in the Dabie orogen. Chem Geol, 2008, 247: 36-65

8 Hermann J. Allanite: Thorium and light rare earth element carrier in subducted crust. Chem Geol, 2002, 192: 289-306

9 Liebscher A, Franz G, Frei D, et al. High-pressure melting of eclogite and the P-T-X history of tonalitic to trondhjemitic zoisite-pegmatites, Münchberg Massif, Germany. J Petrol, 2007, 48: 1001-1019

10 Vielzeuf D, Holloway J R. Experimental determination of the fluid-absent melting reactions in the pelitic system: Consequences for crustal differentiation. Contrib Mineral Petrol, 1988, 98: 257-276

11 Zheng Y F, Xia Q X, Chen R X, et al. Partial melting, fluid supercriticality and element mobility in ultrahigh-pressure metamorphic rocks during continental collision. Earth-Sci Rev, 2011, 107: 342-374

12 Schmidt M W, Poli S. Generation of mobile components during subduction of oceanic crust. Treat Geochem, 2003, 3: 567-591

13 杨经绥, 宋述光, 许志琴, 等. 柴达木盆地北缘早古生代高压-超高压变质带发现典型超高压矿物一柯石英. 地质学报, 2001, 75: 175-179

14 Song S G, Yang J S, Xu Z Q, et al. Metamorphic evolution of the coesite-bearing ultrahigh-pressure terrane in the North Qaidam, Northern Tibet, NW China. J Metamorph Geol, 2003, 21: 631-644 
15 Song S G, Zhang L F, Niu Y L, et al. Geochronology of diamond-bearing zircons from garnet peridotite in the North Qaidam UHPM belt Northern Tibetan Plateau: A record of complex histories from oceanic lithosphere subduction to continental collision. Earth Planet Sci Lett, 2005, 234: 99-118

16 Song S G, Zhang L F, Niu Y L, et al. Evolution from oceanic subduction to continental collision: A case study of the Northern Tibetan Plateau inferred from geochemical and geochronological data. J Petrol, 2006, 47: 435-455

17 Mattinson C G, Wooden J L, Liou J G, et al. Age and duration of eclogite-facies metamorphism, North Qaidam HP/UHP terrane, western China. Amer J Sci, 2006, 306: 683-711

18 Mattinson C G, Wooden J L, Zhang J X, et al. Paragneiss zircon geochronology and trace element geochemistry, North Qaidam HP/UHP terrane, western China. J Asian Earth Sci, 2009, 35: 298-309

19 Zhang G B, Ellis D J, Christy A G, et al. UHP metamorphic evolution of coesite-bearing eclogite from the Yuka terrane, North Qaidam UHPM belt, NW China. Eur J Mineral, 2009, 21: 1287-1300

20 Chen D L, Liu L, Sun Y, et al. Geochemistry and zircon U-Pb dating and its implications of the Yukahe HP/UHP terrane, the North Qaidam, NW China. J Asian Earth Sci, 2009, 35: 259-272

21 Zhang J X, Mattinson C G, Yu S Y, et al. U-Pb zircon geochronology of coesite-bearing eclogites from the southern Dulan area of the North Qaidam UHP terrane, northwestern China: Spatially and temporally extensive UHP metamorphism during continental subduction. J Metamorph Geol, 2010, 28: 955-978.

22 Zhang C, Zhang L F, Roermund H V, et al. Petrology and SHRIMP U-Pb dating of Xitieshan eclogite, North Qaidam UHP. J Asian Earth Sci, 2011, 42: 752-767

23 Zhang C, Roermund H V, Zhang L F, et al. A polyphase metamorphic evolution for the Xitieshan paragneiss of the north Qaidam UHP metamorphic belt, western China: In-situ EMP monazite- and U-Pb zircon SHRIMP dating. Lithos, 2012, 136-139: 27-45

24 张建新, 孟繁聪, 于胜尧, 等. 柴北缘绿梁山高压基性麻粒岩的变质演化历史: 岩石学及锆石 SHRIMP 年代学证据. 地学前缘, 2007, 14: 85-97

25 Xiong Q, Zheng J P, Griffin W L, et al. Zircons in the Shenglikou ultrahigh-pressure garnet peridotite massif and its country rocks from the North Qaidam terrane (western China): Meso-Neoproterozoic crust-mantle coupling and early Paleozoic convergent plate-margin processes. Precambrian Res, 2011, 187: 33-57

26 Zhang J X, Mattinson C G, Meng F C, et al. Polyphase tectonothermal history recorded in granulitized gneisses from the North Qaidam HP/UHP metamorphic terrane, Western China: Evidence from Zircon U-Pb Geochronology. Geol Soc Amer Bull, 2008, 120: 732-749

27 Chen D L, Liu L, Sun Y, et al. Felsic veins within UHP eclogite at Xitieshan in the North Qaidam, NW China: Partial melting during exhumation. Lithos, 2012, 136-139: 187-200

28 孟繁聪, 张建新, 杨经绥. 柴北缘锡铁山早古生代 HP/UHP 变质作用后的构造热事件一花岗岩和片麻岩的同位素与岩石地球化学 证据. 岩石学报, 2005, 21: 45-56

29 孟繁聪, 张建新. 柴北缘绿梁山早古生代花岗岩浆作用与高温变质作用的同时性. 岩石学报, 2008, 24: 1585-1594

30 Gao X Y, Zheng Y F, Chen Y X. Dehydration melting of ultrahigh-pressure eclogite in the Dabie orogen: Evidence from multiphase solid inclusions in garnet. J Metamorph Geol, 2012, 30: 193-212

31 Zhao Z F, Zheng Y F, Chen R X, et al. Element mobility in mafic and felsic ultrahigh-pressure metamorphic rocks during continental collision. Geochim Cosmochim Acta, 2007, 71: 5244-5266

32 Larikova T, Holzheid A, Kegler P. Geochemistry of tonalites formed by parting melting of eclogites: Experimental modelling. Mineral Mag Goldschm Conference Abs, 2011, 1270

33 Shatsky V S, Jagoutz E, Sobolev N V. Geochemistry and age of ultrahigh pressure metamorphic rocks from the Kokchetav massif (Northern Kazakhstan). Contrib Mineral Petrol, 1999, 137: 185-205

34 Franz G, Smelik G. Zoisite-clinozoisite bearing pegmatites and their importance for decompressional melting in eclogites. Eur J Mineral, 1995, 7: 1421-1436 